\title{
THE RELATIVE CONNECTIVITIES OF SYMMETRIC PRODUCTS*
}

\section{BY MOSES RICHARDSON}

1. Introduction. The topology of the domain of discontinuity of a finite group of transformations operating on a complex, and, in particular, the topology of symmetric product complexes, has been studied by P. A. Smith $\dagger$ and the author. $f$ Following a suggestion made by Morse, $\S$ we obtain in this note explicit formulas for the so-called relative connectivities of the symmetric product of a complex in terms of its mod 2 Betti numbers, and we discuss an application of this result to the theory of critical chords. First, however, we derive a more general result of which the formulas for the relative connectivities of symmetric products is a special case. The methods used here follow closely those of S.

2. Definitions and Preliminary Theorems. For proofs or fuller discussion of statements made in this section, the reader is referred to $\mathrm{S}$ or $\mathrm{R}$.

Let $K$ be a simplicial $n$-complex. $\|$ Let $T$ be a topological involution such that (a) $T$ carries $m$-simplexes of $K$ into $m$ simplexes of $K$; (b) if a simplex of $K$ is invariant, it is pointwise invariant.

The invariant simplexes of $K$ form a subcomplex $K^{0}$, and the non-invariant simplexes can be grouped in pairs so that each member of a pair is transformed into the other member by $T$. Thus the $m$-simplexes of $K$ can be renamed $E_{m}{ }^{i}, \bar{E}_{m}{ }^{i}, E_{m}{ }^{0 j}$, where $\bar{E}_{m}^{i}=T E_{m}^{i}$, and $E_{m}{ }^{j}$ is a simplex of $K^{0}$. If $\Upsilon C=t_{i} E_{m}{ }^{i}$ is a chain of

* Presented to the Society, February 23, 1935.

$\dagger$ P. A. Smith, The topology of involutions, Proceedings of the National Academy of Sciences, (1933), pp. 612-618. (Denoted hereafter by S.)

$\ddagger \mathrm{M}$. Richardson, On the homology characters of symmetric products, Duke Mathematical Journal, vol. 1 (1935), pp. 50-69. (Denoted hereafter by R.)

$\S \mathrm{M}$. Morse, The Calculus of Variations in the Large, Colloquium Publications of this Society, vol. 18, 1934, p. 191. (Denoted hereafter by M.)

\| Our general topological terminology and notation is that of S. Lefschetz, Topology, Colloquium Publications of this Society, vol. 12, 1930.

I A repeated index indicates summation. 
$K$, we define $T C$ to be the chain $\bar{C}=t_{i} \bar{E}_{m}{ }^{i}$. The involution $T$ preserves bounding relations. $\dagger$

We consider only mod 2 topology; all homologies and equations are understood to be homologies and congruences mod 2.

A chain $X$ of $K$ is called invariant if $X=\bar{X}$. In particular, if every simplex occurring in a chain with a non-zero coefficient belongs to $K^{0}$, we attach a zero to the chain-symbol, as $X^{0}$. If no simplex of $K^{0}$ occurs in a chain with a non-zero coefficient, we attach an asterisk to the chain-symbol, as $X^{*}$. Every invariant chain can be written in the form $X^{*}+\bar{X}^{*}+X^{0}$. If an invariant cycle $\Gamma$ is the boundary of an invariant chain, we write $\Gamma \cong 0$. These special homologies obey the same formal rules as ordinary homologies.

We choose a base for homology of type $\Gamma^{i}, \bar{\Gamma}^{i}, D^{i}$ for each dimension. $\ddagger$ We consider only the case in which (A) $D_{m}{ }^{j}+\bar{D}_{m}{ }^{j}$ $\cong 0$ for every $m>0$ and every $j$. In this case we can and do replace the $D_{m}^{i}$ in the base by invariant cycles ${ }^{i} \Delta_{m}$.

We now construct the sequences

$$
{ }^{i} \Delta_{m}^{m},{ }^{i} \Delta_{m-1}^{m}, \cdots,{ }^{i} \Delta_{r}^{m}, \quad(r=r(m, i) \geqq-1),
$$

where ${ }^{i} \Delta_{q}{ }^{m}={ }^{i} X_{q}{ }^{m}+{ }^{i} \bar{X}_{q}{ }^{m}$, and $F\left({ }^{i} X_{q}{ }^{m}\right)={ }^{i} \Delta_{q-1}^{m}$, (for $q=m, m-1$, $\cdots, r+1)$, and ${ }^{i} \Delta_{r}^{m}={ }^{i} X_{r}^{m}+{ }^{i} \bar{X}_{r}^{m}+{ }^{i} X_{r}^{0 m}$, (for $r \geqq 0$ ), where the ${ }^{i} X_{r}^{0 m}$ are cycles.|| We consider only the case where (B) the cycles ${ }^{i} X_{r}^{0 m}$ are independent with respect to homologies on $K^{0}$. We shall need the following lemmas. II

(2) If $C+\bar{C}+C^{0} \cong 0$, then $C^{0} \sim 0$ on $K^{0}$.

(3) The cycles $\Gamma_{q}^{i}+\bar{\Gamma}_{q}^{i},{ }_{i} \Delta_{q}{ }^{m},(q>0)$, are independent with respect to $\cong$.

(4) If (A) and (B) hold, every cycle of the form $C_{q}+\bar{C}_{q},(q>0)$, is $\cong$ to a linear combination of cycles $\Gamma_{q}^{i}+\bar{\Gamma}_{q}^{i},{ }^{i} \Delta_{q}{ }^{m}$.

With the simplexes $E_{m}{ }^{i}$ and $\bar{E}_{m}{ }^{i}$ we associate $\nmid$ a simplex $e_{m}{ }^{i}$, and we write $\wedge E_{m}{ }^{i}=\wedge \bar{E}_{m}{ }^{i}=e_{m}{ }^{i}$. If $C=t_{i} E_{m}{ }^{i}$, we define $\wedge C$ to be the chain $c=t_{i} e_{m}{ }^{i}$. The totality of simplexes $e_{m}{ }^{i}$ constitutes

$\dagger \mathrm{R}, \S 1$.

$\ddagger S, \S 1$.

$\S \mathrm{S}, \mathrm{p} .614$.

$\|$ S, p. 614.

If $\mathrm{S}, \mathrm{pp} .613-615$.

$\dagger$ The material in this paragraph is fully discussed in $R, \S 2$. 
an $n$-complex $k=\wedge K$, say. In particular, the simplexes $e_{m}^{0 i}$ $=\wedge E_{m}^{0 i}$ constitute a subcomplex $k^{0}=\wedge K^{0}$, say. If $e=\wedge E$ is a simplex of $k$, we write $\wedge^{\prime} e=E+\bar{E}$. If $c=t_{i} e_{m}{ }^{i}$, we define $\wedge^{\prime} c$ to be the chain $t_{i} \wedge^{\prime} e_{m}^{i}$. Both $\wedge$ and $\wedge^{\prime}$ preserve bounding relations. We shall use large or small letters for chains of $K$ or $k$, respectively. In particular, a symbol like $x^{0}$ will denote a chain of $k^{0}$, and a symbol like $x^{*}$ will denote a chain in which no cell of $k^{0}$ occurs with a non-zero coefficient.

3. The Topology of $k \bmod k^{0}$. We shall now determine the Betti numbers $R_{q}\left(k ; k^{0}, 2\right)$. A chain whose boundary is a chain of $k^{0}$, that is, a cycle $\bmod k^{0}$, shall be called a relative cycle.

(5) If $c+x^{0} \rightarrow 0$, then $c$ is a relative cycle.

Proof. Let $F(c)=y^{*}+y^{0}$ and $F\left(x^{0}\right)=z^{0}$. Since $F\left(c+x^{0}\right)$ $=y^{*}+y^{0}+z^{0}=0$, we have $y^{*}=0$. Hence $c \rightarrow 0 \bmod k^{0}$.

(6) If $c+x^{0} \sim 0$, then $c \sim 0 \bmod k^{0}$.

Proof. There exists a chain $d$ such that $d \rightarrow c+x^{0}$. Thus $d \rightarrow c \bmod k^{0}$.

(7) If $\gamma$ is a relative cycle, then $\wedge^{\prime} \gamma$ is a cycle.

Proof. Since $\gamma \rightarrow x^{0}$, we have $\Lambda^{\prime} \gamma \rightarrow \wedge^{\prime} x^{0}=X^{0}+\bar{X}^{0}=2 X^{0}=0$.

(8) If $\gamma \sim 0 \bmod k^{0}$, then $\wedge^{\prime} \gamma \cong 0$.

Proof. Since there exists a chain $c$ such that $c \rightarrow \gamma+x^{0}$, we have $\wedge^{\prime} c \rightarrow \wedge^{\prime} \gamma+\wedge^{\prime} x^{0}$. But $\wedge^{\prime} x^{0}=0$. It is obvious that $\wedge^{\prime} c$ and $\wedge^{\prime} \gamma$ are invariant.

(9) If $c$ is a relative cycle, we can write $\wedge^{\prime} c$ in the form $C+\bar{C}$ where $\wedge C=c$.

Proof. Let $c=t_{i} e_{m}^{i}+u_{i} e_{m}^{0 i}$. We have only to let $C=t_{i} E_{m}{ }^{i}$ $+u_{i} E_{m}{ }^{0 i}$.

(10) If $C+\bar{C} \cong 0$, then $\wedge C$ is a relative cycle and $\wedge C \sim 0 \bmod k^{0}$.

Proof. By hypothesis, $H+\bar{H} \rightarrow C+\bar{C}$. Let $F(H)=C+X$. Then $X+\bar{X}=0$. Hence $X=X^{*}+\bar{X}^{*}+X^{0}$. Therefore,

$$
\wedge H \rightarrow \wedge C+\wedge X=\wedge C+2 \wedge X^{*}+\wedge X^{0}=\wedge C+\wedge X^{0} .
$$

Thus $\wedge C+\wedge X^{0} \rightarrow 0$, and by (5), $\wedge C$ is a relative cycle. Since $\wedge H \rightarrow \wedge C+\wedge X^{0} \sim 0$, we have $\wedge C \sim 0 \bmod k^{0}$, by (6). 
(11) For $q \geqq r(m, i)+1, \wedge\left({ }^{i} X_{q}{ }^{m}\right)$ is a relative cycle, say ${ }^{i} \xi_{q}{ }^{m}$, and $\wedge^{\prime}\left(i \xi_{q}^{m}\right)={ }^{i} \Delta_{q}{ }^{m}$.

Proof. If $q \geqq r(m, i)+2$, then, since ${ }^{i} X_{q}{ }^{m} \rightarrow^{i} \Delta_{q-1}^{m}$, we have

$$
\wedge\left({ }^{i} X_{q}^{m}\right) \rightarrow \wedge\left({ }^{i} \Delta_{q-1}^{m}\right)=\wedge\left({ }^{i} X_{q-1}^{m}+{ }^{i} \bar{X}_{q-1}^{m}\right)=0 .
$$

Therefore, $\wedge\left({ }^{i} X_{q}{ }^{m}\right)={ }^{i} \xi_{q}{ }^{m}$ is an absolute cycle. If $q=r(m, i)+1$, we have

$$
\begin{aligned}
\wedge\left({ }^{i} X_{q}^{m}\right) \rightarrow \wedge\left({ }^{i} \Delta_{q-1}^{m}\right) & =\wedge\left({ }^{i} X_{r}^{m}+{ }^{i} \bar{X}_{r}^{m}+{ }^{i} X_{r}^{0 m}\right) \\
& =\wedge\left({ }^{i} X_{r}^{0 m}\right)=0 \bmod k^{0} .
\end{aligned}
$$

Thus, in this case, ${ }^{i} \xi_{q}{ }^{m}=\wedge\left({ }^{i} X_{q}{ }^{m}\right)$ is a relative cycle. In either case we have $\wedge^{\prime}\left({ }^{i} \xi_{q}{ }^{m}\right)={ }^{i} X_{q}{ }^{m}+{ }^{i} \bar{X}_{q}{ }^{m}={ }^{i} \Delta_{q}{ }^{m}$.

Let $\gamma_{q}^{i}=\wedge \Gamma_{q}^{i}$.

(12) The relative cycles $\gamma_{q}^{i},{ }^{i} \xi_{q}{ }^{m},(q \geqq r(m, i)+1>0)$, are independent with respect to homology $\bmod k^{0}$.

Proof. Suppose there were a non-trivial homology

$$
x_{i m}^{i} \xi_{q}^{m}+y_{i} \gamma_{q}^{i} \sim 0 \bmod k^{0} \text {. }
$$

By (8) we have $\wedge^{\prime}\left(x_{i m}^{i} \xi_{q}^{m}+y_{i} \gamma_{q}^{i}\right) \cong 0$. Thus, by (11),

contradicting (3).

$$
x_{i m}^{i} \Delta_{q}^{m}+y_{i}\left(\Gamma_{q}^{i}+\bar{\Gamma}_{q}^{i}\right) \cong 0
$$

(13) Every relative $q$-cycle of $k$ is homologous $\bmod k^{0}$ to a linear combination of the $\gamma_{q}^{i}$ and ${ }^{i} \xi_{q}{ }^{m},(q \geqq r(m, i)+1>0)$.

Proof. Let $\gamma$ be an arbitrary relative $q$-cycle of $k$. Let $\wedge^{\prime} \gamma=\Gamma+\bar{\Gamma}$, where $\wedge \Gamma=\gamma$, by (9). By (7), $\wedge^{\prime} \gamma$ is a cycle. Therefore, by (4),

$$
\Gamma+\bar{\Gamma} \cong x_{i}\left(\Gamma_{q}^{i}+\bar{\Gamma}_{q}^{i}\right)+y_{i m}^{i} \Delta_{q}^{m} .
$$

Now we shall show that $y_{i m}=0$ whenever $r(m, i)=q$. For, if some $y_{i m} \neq 0$, then (14) would be of the form

$$
Y+\bar{Y}+z_{i m}\left({ }^{i} X_{q}^{m}+{ }^{i} \bar{X}_{q}^{m}+{ }^{i} X_{q}^{0 m}\right) \cong 0
$$

where some $z_{i m} \neq 0$. This implies that $z_{i m}{ }^{i} X_{q}^{0 m} \sim 0$ on $K^{0}$, by (2). But this contradicts (B). Thus, (14) has the form

$$
\Gamma+\bar{\Gamma}+x_{i}\left(\Gamma_{q}^{i}+\bar{\Gamma}_{q}^{i}\right)+y_{i m}\left({ }^{i} X_{q}^{m}+{ }^{i} \bar{X}_{q}^{m}\right) \cong 0,
$$


where $q \geqq r(m, i)+1$. Let $C=\Gamma+x_{i} \Gamma_{q}^{i}+y_{i m}{ }^{i} X_{q}^{m}$. Then $C+\bar{C} \cong 0$, and, by $(10)$, we have $\wedge C \sim 0 \bmod k^{0}$, or

$$
\gamma+x_{i} \gamma_{q}^{i}+y_{i m}^{i} \xi_{q}^{m} \sim 0 \bmod k^{0} \text {. }
$$

This proves the theorem.

By (12) and (13), the relative cycles ${ }^{i} \xi_{q}{ }^{m}, \gamma_{q}^{i},(q \geqq r(m, i)+1$ $>0$ ), constitute a base for relative $q$-cycles of $k$ with respect to homology $\bmod k^{0}$. Let $R_{q}^{\Gamma}$ be the number of cycles $\Gamma_{q}^{i}$, and let $Q_{q}$ be the number of $q$-cycles ${ }^{i} \Delta_{q}{ }^{m}$ satisfying the relation $r(m, i)+1 \leqq q$. We have proved the following theorem.

THEOREM 1. If the hypotheses (a), (b), (A), and (B) are fulfilled, then $R_{q}\left(k ; k^{0}, 2\right)=R_{q}^{\Gamma}+Q_{q},(q>0)$.

4. Symmetric Products. Let $K_{2 n}=K_{n} \times K_{n}$ be the complex $K$ of the preceding sections. Let $T$ be the involution which interchanges the points $P \times Q$ and $Q \times P$ of $K_{2 n}$. A simplicial subdivision of $K_{2 n}$ satisfying (a) and (b) of $\$ 2$ can be found. $\dagger$ Of course, $\wedge K_{2 n}=k_{2 n}$ is the 2 -fold symmetric product of $K_{n}$. We can choose bases for homology on $K_{2 n}$ of the $\Gamma, \bar{\Gamma},{ }^{j} \Delta$ type here required. $\ddagger$ The cycles ${ }^{i} \Delta_{q}$ occur only in even dimensions. It has been shown that the sequences (1) can be constructed so that $r(2 h, i)=h$ for all $i$, and so that (A) and (B) are fulfilled. $\$$ Therefore we may apply Theorem 1 .

Now let $R_{2 s}^{\Delta}=R_{s}\left(K_{n}, 2\right)$ for $s \leqq n$ and $R_{2 s}^{\Delta}=0$ for $s>n$. Then it is easily seen that $Q_{1}=0$ and

$$
Q_{q}=R_{2 t}^{\Delta}+R_{2(t+1)}^{\Delta}+\cdots+R_{2(q-1)}^{\Delta}, \quad(q>1),
$$

where $t=[(q+1) / 2]$, since the lowest dimension $2 m$ to yield cycles ${ }^{i} \Delta_{q}{ }^{2 m}$ is either $2 m=q$ or $2 m=q+1$. Thus by Theorem 1 , we have the following result.

TheOREM 2. For the symmetric product $k_{2 n}$ of $K_{n}$ we have $R_{1}\left(k_{2 n} ; k_{n}^{0}, 2\right)=R_{1}^{\Gamma}$ $R_{q}\left(k_{2 n} ; k_{n}^{0}, 2\right)=R_{q}^{\Gamma}+R_{2 t}^{\Delta}+R_{2(t+1)}^{\Delta}+\cdots+R_{2(q-1)}^{\Delta}, \quad(q>1)$, where $t=[(q+1) / 2]$, and where

$\dagger \mathrm{R}, \S 5$.

$\ddagger R$, p. 57.

$\S \mathrm{R}, \mathrm{pp} .64-65$. 


$$
\stackrel{\Gamma}{R_{q}}=\frac{1}{2}\left[R_{q}\left(K_{2: \imath}, 2\right)-R_{q}^{\Delta}\right]
$$

if $q$ is even, and

$$
R_{q}^{\Gamma}=\frac{1}{2} R_{q}\left(K_{2 n}, 2\right)
$$

if $q$ is odd.

Of course, if $K_{n}$ is connected, so is $k_{2 n}$; hence $R_{0}\left(k_{2 n} ; k_{n}^{0}, 2\right)=0$ in this case. The numbers $R_{q}\left(k_{2 n} ; k_{n}^{0}, 2\right)$ have been called relative connectivities by Morse, $\uparrow$ who proved that they are finite. $\ddagger$ This result is of course implied by our formulas.

Example 1 . Let $K_{n}$ be an $n$-sphere. Then $R_{n}^{\Gamma}=R_{2 n}^{\Delta}=1$, while all the other $R^{\Gamma}$, s and $R^{\Delta}$ 's, are zero. From our formulas, we obtain for the relative connectivities of $k_{2 n}$,

$$
\begin{aligned}
& R_{0}=R_{1}=\cdots=R_{n-1}=0 \\
& R_{n}=R_{n}^{\Gamma}=1 \\
& R_{n+1}=R_{2(q-1)}^{\Delta}=R_{2 n}^{\Delta}=1 \\
& R_{n+2}=R_{2(q-2)}^{\Delta}=R_{2 n}^{\Delta}=1 \\
& \cdot \cdot \cdot \cdot \cdot \cdot \cdot \cdot \cdot \cdot \cdot \\
& R_{2 n}=R_{2 t}^{\Delta}=R_{2 n}^{\Delta}=1 .
\end{aligned}
$$

The values of the relative connectivities for this example were worked out by Morse $\S$ by special methods involving the critical chords of an $n$-ellipsoid.

ExAmple 2. Let $K_{n}$ be an orientable surface of genus $p$. Then the relative connectivities of the symmetric product $k_{2 n}$ are $R_{0}=0, R_{1}=2 p, R_{2}=2 p^{2}+p+1, R_{3}=2 p+1, R_{4}=1$.

5. Application to the Theory of Critical Chords.\| The chief results concerning critical chords are as follows. Let $R$ be a regular, analytic Riemannian $n$-manifold lying in a euclidean $(n+1)$ space, such that $R$ is homeomorphic to a simplicial $n$-complex $K_{n}$. Then the symmetric product of $R$ is evidently homeo-

$\dagger \mathrm{M}, \mathrm{p} .182$.

$\ddagger$ M, pp. 182-183.

$\S \mathrm{M}$, Theorem 11.3, p. 191.

|| For definitions and proofs required in this section see M, pp. 181-191. 
morphic to the symmetric product $k_{2 n}$ of $K_{n}$. Let $R_{0}, R_{1}, \cdots$, $R_{2 n}$ be the relative connectivities of $k_{2 n}$. Then the sums $M_{i}$ of the type numbers of the critical sets of chords of $R$ and the numbers $R_{i}$ satisfy the relations

$M_{0} \geqq R_{0}, M_{0}-M_{1} \leqq R_{0}-R_{1}, M_{0}-M_{1}+M_{2} \geqq R_{0}-R_{1}+R_{2}$,

$M_{0}-M_{1}+\cdots+(-1)^{2 n} M_{2 n}=R_{0}-R_{1}+\cdots+(-1)^{2 n} R_{2 n} . \dagger$

A simple corollary of this theorem is this: If the critical chords of $R$ are all non-degenerate, there exist at least $R_{i}$ such chords of index $\ddagger$.

Our Theorem 2 enables us to obtain the values of the relative connectivities $R_{i}$ of $k_{2 n}$ when the mod 2 Betti numbers of $R$ are known. Thus the above theorem and its corollary can be used to obtain numerical information concerning the critical chords of any $R$ whose mod 2 Betti numbers are known. This makes available a wide class of examples. For instance, the corollary of M, p. 191 follows at once from the above corollary and our Example 1, \$4.

As a further example, let $R$ be any regular, analytic image of an orientable surface of genus $p$. Then, from Example 2, $\$ 4$, and the above corollary, we obtain the result that, if the extremal chords of $R$ are all non-degenerate, then, among these extremal chords there must be $2 p^{2}+5 p+3$ extremal chords of the following description: $2 p$ extremal chords of index $1,2 p^{2}+p+1$ extremal chords of index $2,2 p+1$ extremal chords of index 3 , and 1 extremal chord of index 4 . In the degenerate case, the same result holds provided each critical set of chords is counted according to its type numbers.

Brooklyn College

$\dagger \mathrm{M}$, Theorem 11.1, p. 185.

$\ddagger$ M, p. 185. 